\title{
A Novel Method of Piggyback Jejunal Interposition Reconstruction Single-Tract Reconstruction After Proximal Gastrectomy for Siewert II and III Adenocarcinoma of the Esophagogastric Junction with a Diameter $<4 \mathrm{~cm}$
}

\author{
Yuchao Ma \\ Shanxi Medical University \\ Jianhong Dong ( $\square$ asdjh666@163.com ) \\ Shanxi Provincial Cancer Hospital

\section{Baoqing Ren} \\ Shanxi Provincial People's Hospital \\ Wanhong Zhang \\ Shanxi Provincial Cancer Hospital \\ Liqiang Lei \\ The Second Hospital of Shanxi Medical University \\ Ning Xu \\ Linfen Central Hospital \\ Linjie Li \\ Shanxi Provincial Cancer Hospital
}

\section{Research Article}

Keywords: Proximal gastrectomy, Single-tract reconstruction, Adenocarcinoma of the esophagogastric junction (AEG) , Postoperative reflux

Posted Date: December 6th, 2021

DOI: https://doi.org/10.21203/rs.3.rs-1131676/v1

License: (c) (1) This work is licensed under a Creative Commons Attribution 4.0 International License.

Read Full License 


\section{Abstract}

Background Digestive tract reconstruction after proximal gastrectomy (PG) has been extensively discussed. Herein, we introduce a novel method of PG with piggyback jejunal interposition reconstruction single-tract reconstruction (PJIRSTR) for Siewert II and III adenocarcinoma of the esophagogastric junction (AEG) with a diameter $<4 \mathrm{~cm}$, and investigate its safety, practicability, and short-term and longterm clinical outcomes of this procedure.

Method The clinical data of 33 patients with Siewert II or Siewert III AEG who underwent PJIRSTR in Shanxi Cancer Hospital from July 2013 to November 2016 were retrospectively reviewed. Data of clinicopathologic characteristics, postoperative and surgical outcomes, and follow-up findings, especially postoperative reflux esophagitis and postoperative reflux symptoms, were analyzed.

Results The mean operation time was $136.7 \pm 22.4$ (range: $110-180$ ) min, including $32.3 \pm 5.0$ (range: 26 45) min of the digestive tract reconstruction; the mean estimated blood loss was $87.6 \pm 18.1 \mathrm{~mL}$; the mean number of dissected lymph nodes was $14.7 \pm 5.1$; and the mean duration of postoperative hospitalization was $7.5 \pm 1.2$ days. The early complication rate was $9 \%(n=3)$, including one case each of anastomotic bleeding, incision infection, and ileus. The late complication rate was $6 \%(n=2)$ : both patients had gastroesophageal reflux symptoms (Visick grade II), but only one patient had Los Angeles grade B reflux esophagitis by endoscopy.

Conclusion PJIRSTR is a safe, feasible, and innovative reconstruction method after PG for patients with Siewert II and III AEG with a diameter $<4 \mathrm{~cm}$. Furthermore, it has excellent efficiency in terms of preventing reflux symptoms and reflux esophagitis after surgery.

\section{Introduction}

In the past few decades, the incidence of proximal gastric cancer has significantly increased worldwide[13]; accordingly, extensive research on adenocarcinoma of the esophagogastric junction (AEG) is also being carried out. For AEG treatment, total gastrectomy (TG) is usually preferred considering the oncological safety and the high incidence of complications such as reflux esophagitis and anastomotic strictures after proximal gastrectomy (PG). However, some studies have shown that PG and TG have similar 3-year survival rates in early AEG[4-6], and PG has been reported to be oncologically safe for patients with T2/T3 proximal gastric cancer[7]. In addition, PG has significant advantages over TG with respect to postoperative nutritional status [5-6]. However, reflux esophagitis and reflux symptoms after PG have plagued surgeons for many years. Although there are various digestive tract reconstruction methods after PG to solve this problem, no ideal method has found wide application so far owing to the inefficient reflux prevention or the technique not gaining adequate popularity for varied reasons.

Herein, we introduce a novel method called piggyback jejunal interposition reconstruction single-tract reconstruction (PJIRSTR) that can effectively prevent postoperative reflux symptoms and reflux 
esophagitis. To our knowledge, PJIRSTR in Siewert II and III AEG with a diameter $<4 \mathrm{~cm}$ has not yet been reported in detail.

\section{Patient And Methods}

\section{Patients}

From July 2013 to November 2016, 33 patients with Siewert II or III AEG who underwent PJIRSTR at Shanxi Cancer Hospital were selected. The inclusion criteria were as follows: (1) age range between 45 and 70 years; (2) Siewert II or III AEG confirmed by postoperative pathological findings; (3) tumor diameter $<4 \mathrm{~cm}$ without distant metastasis, and invasion of the lower esophagus $<1 \mathrm{~cm}$; and (4) no vital organ dysfunction and no surgical contraindications. Before the surgery, a routine gastroscopy was performed to assess whether the patients had reflux esophagitis and its severity. Endoscopic findings were classified according to the Los Angeles Classification. Furthermore, the Visick score also was routinely used for all patients to evaluate gastroesophageal reflux symptoms.

\section{Surgical procedure}

After the patients were admitted to the hospital, relevant examinations and tests were completed, then the operation was performed after excluding contraindications. The same surgeon and their surgical team carried out all operations.

\section{PG+ esophagojejunal Roux-en-Y anastomosis}

For PJIRSTR, the esophagojejunal Roux-en-Y anastomosis was first performed, and the jejunal loop was about $30 \mathrm{~cm}$. On this basis, an anastomosis of the remnant stomach and jejunum was performed at a suitable position of the jejunal loop, which resembled the jejunum carrying the remnant stomach on its back.

After induction of general anesthesia, the patients were placed in the supine position. The entire procedure was carried out via a transabdominal approach, with a midline incision made to the upper abdomen. After entering the abdominal cavity, the greater and lesser omentum were released. According to the Third and Fourth edition Japanese gastric cancer treatment guidelines, D1+ or D2 lymph node dissection was performed based on the size, location, and degree of esophageal invasion of the tumor. The digestive tract was reconstructed after PG, and at least half of the stomach was preserved. The jejunum and its mesangial blood vessels were cut at about $20-25 \mathrm{~cm}$ from the Treitz ligament, and the distal jejunum was raised to the end of the esophagus in front or behind the colon. The circular stapler was used to perform an end-to-side anastomosis between the esophagus and distal jejunum. The stump of the jejunum was closed with a linear closure device, and the length of the blind end $<3 \mathrm{~cm}$. Lateral anastomosis between the proximal and distal jejunum was performed about $30 \mathrm{~cm}$ away from the distal end of the esophageal jejunum anastomosis. The residual end was closed with a linear cutting closure device, and the length of the blind end was $\leq 3 \mathrm{~cm}$. 


\section{PJIRSTR was performed after PG}

The key of PJIRSTR: (i) The side-to-side anastomosis was performed between the anterior wall of the remnant stomach and the jejunum while keeping the anastomosis $3-5 \mathrm{~cm}$ away from the stump of the remnant stomach, which formed a structure of "artificial gastric fundus" and anastomosis size was about $4-5 \mathrm{~cm}$. (ii) After anastomosis was completed, the angle formed by the long axis of the remnant stomach and the long axis of the jejunum should be $<75^{\circ}$. (iii) The length of the interposition jejunum remains $12-$ $15 \mathrm{~cm}$. (iv) The jejunum was closed with a linear closure device at $<3 \mathrm{~cm}$ below the anastomosis of the residual stomach jejunum to completely block the jejunum content channel and complete the PJIRSTR (Figure 1).

\section{Postoperative care}

All patients had indwelling nutrition tubes during surgery to provide nutritional support after surgery. Enteral nutrition support was provided for 1 week from day 1 after surgery; after 1 week, oral nutrition supplementation was started. The drainage tube was pulled out depending on each patient's condition. If the laboratory tests (blood test, liver, and kidney function) and upper gastrointestinal contrast 7-10 days after the operation were normal, and patients exhibited no abdominal pain or other discomfort, they were usually discharged from the hospital about 1 weeks after surgery.

\section{Evaluation of the clinical indicators}

Surgical outcomes, postoperative complications, and patient's surgical parameters (e.g., age, sex, weight, body mass index [BMI], histological type, pathologic findings, number of retrieved and metastatic [LNs]) were recorded. Early complications (<30 days) including anastomotic bleeding, anastomosis leakage, anastomotic stenosis, incision infection, ileus, and organ dysfunction were analyzed. As for the late complications (>30 days), it mainly referred to reflux esophagitis, reflux symptoms, recannulation of intestinal tract, and changes in nutritional indicators, which were also analyzed. Visick scores were calculated for all patients via telephonic follow-up at postoperative 3, 6, and 12 months; all patients returned to the hospital for a gastroscopic review at postoperative 6 and 12 months. Gastroesophageal reflux symptoms were classified by Visick score, and reflux esophagitis was classified by the Los Angeles classification according to the endoscopy findings. In addition, patients were required to return to the hospital for a review of body weight and levels of hemoglobin, total serum protein, and serum albumin levels to assess changes in nutritional indicators at postoperative 3,6 , and 12 months.

\section{Statistical methods}

Statistical analysis was performed using SPSS 26.0 software. All values were expressed as the mean \pm standerd deviation (SD). The difference in complication rates and operative data between the Phase I study and the current study were analyzed using the Chi-square test and T-test, respectively. Paired-samples t-test was used to analyze the nutritional indicators. $P<0.05$ was considered statistically significant. 


\section{Results}

\section{Patient characteristics}

The baseline characteristics of patients are summarized in Table 1. The study population comprised 29 $(87.9 \%)$ male and $4(12.1 \%)$ female patients (mean age: 60.9 [range, 46-72] years and mean BMI:

$24.1 \pm 2.6$ [range, $18.25-28.26$ ] kg/m²). There were $9(27.3 \%)$ patients who had comorbidities. A history of previous abdominal operation did not exist in 33 patients. No patient had reflux esophagitis per preoperative endoscopic evaluation. Further, 3 (9.1\%) patients showed Visick grade Il gastroesophageal reflux symptoms before surgery.

\section{Surgical outcomes and pathological findings}

All surgeries were open surgery via the abdominal transhiatal $(\mathrm{TH})$ approach. Four patients underwent D1+ lymphadenectomy, and all others underwent D2 lymphadenectomy. The surgical outcomes of 33 patients are presented in Table 2. The mean operative time was 136.7 \pm 22.4 (range: 110-180) min, including $32.3 \pm 5.0$ (range: $26-45$ ) min of digestive reconstruction. The intraoperative blood loss was $87.6 \pm 18.1$ (range: $60-120$ ) $\mathrm{mL}$. The tumor size was $3.06 \pm 0.84$ (range: $1-4$ ) $\mathrm{cm}$, and R0 resection was performed in all patients. There were no deaths or serious complications during the operation.

Pathological findings are shown in Table 3. According to the TNM staging (8th edition AJCC), three, three, five, two, one, eight, and 12 cases were staged IA, IB, IC, IIB, IIIA, IIIB, and IVA, respectively. Histologic types comprised 21 moderately well-differentiated cases and 12 poorly differentiated cases. The upper and lower resection margins of all patients were negative at postoperative pathological examination. The average number of LNs dissected was $14.7 \pm 5.1$ (range: 9-27) and the mean number of metastatic LNs was 2.6 \pm 3.4 (range: $0-12$ ).

\section{Early complications and late complications}

The overall early complication rate was $9 \%(n=3)$, including one each of anastomotic bleeding, incision infection, and ileus that improved after conservative management without the need for reoperation. Anastomotic bleeding and incision infection were managed and improved by conservative treatment. The late complication rate was $6 \%(n=2)$ : both patients had Visick grade II reflux symptoms (at postoperative 3 and 6 months, respectively); only one patient showed Los Angeles grade B reflux esophagitis upon endoscopy. Both patients regularly took proton-pump inhibitors (PPIs) and followed the doctor's advice and were relieved of the reflux symptoms upon review. The results of gastroscopy are shown in Figure 2. All patients showed promising imaging results upon upper gastrointestinal angiography 7-10 days after the operation with no anastomotic stenosis and no recanalization at the jejunal block. Furthemore, no recannulation of intestinal tract was observed during follow-up period. Figure 3 shows the post PG upper gastrointestinal radiography findings, using PJIRSTR.

\section{Recurrence and survival data}


The median follow-up period was 60.7 (range: $27-90.8$ ) months. One patient with pathological stage IVA was diagnosed with gastric stump cancer at postoperative 25 months and underwent re-surgery. The patient subsequently died 16 months after the second operation. The 3-year survival rate of the 33 patients was $90.9 \%$. Adjuvant chemotherapy was performed in 23 patients according to the Sixth and Seventh editions of AJCC/UICC.

\section{Nutritional indices}

Clinicians tended to use body weight, serum albumin, hemoglobin, and total serum protein as measures of nutritional status. Therefore, corresponding indicators were monitored at preoperative and postoperative 3,6 , and 12 months. The results of nutritional indices are shown in Figure 4 . All indicators decreased significantly 3 month after surgery compared with those before the operation $(A: t=5.058, p<$ $0.0001 ; B: t=10.206, p<0.0001 ; C: t=6.33, p<0.0001 ; D: t=12.609, p<0.0001)$. However, most patients, in addition to body weight, the other three indicators returned to normal ranges at postoperative12 months. The average weight loss was $13.5 \%, 11.8 \%$, and $10 \%$ at postoperative 3,6 , and 12 months, respectively.

\section{Comparison with previous study}

We compared the major surgical results and follow-up results of this study with the Phase I clinical study, and the results showed that postoperative hospital stays was significantly shorter $(p<0.05)$ and there was no significant difference in early complications and stenosis $(p>0.05)$. However, the incidence of reflux symptoms and reflux esophagitis was significantly decreased in the current study. (Table 4)

\section{Discussion}

In this study, we analyzed the results of the surgical outcomes of 33 patients who had Siewert II and III AEG with a diameter $<4 \mathrm{~cm}$ and underwent PJIRSTR. The PJIRSTR technique was first proposed by our research team in 2016[8]. After years of improvement and exploration, we believe that this technique has achieved very promising results. To our knowledge, this is the first detailed description of the PJIRSTR technique. Based on the results of our analysis, PJIRSTR effectively solves the problem of reflux after PG. It is an acceptable treatment method for the Siewert II and III AEG with a diameter $<4 \mathrm{~cm}$.

According to the Japanese gastric cancer treatment guidelines (Fifth edition), for early AEG, if more than half of the stomach after RO resection can be preserved, $\mathrm{PG}$ can be selected as the surgical method[9]. However, it is still unclear whether PG can be performed for advanced AEG. Sugoor et al.[10] reported that as long as sufficient surgical resection margins are ensured and enough remnant stomach is preserved, PG can be selected even for advanced proximal-third gastric cancer. Haruta et al.[11] believed that patients with advanced AEG whose tumor length is $<4 \mathrm{~cm}$ could undergo PG.

As a functional preservation surgery, PG has some irreplaceable advantages compared with TG. First, PG ensures that food stays in the residual stomach for preliminary digestion, and then further digestion through the duodenum, which is conducive to full absorption in the jejunum and the nutritional status of 
patients after surgery. Second, PG provides a better pathway for further endoscopic treatment such as endoscopic retrograde cholangiopancreatography (ERCP) than TG. A recently conducted multi-center, prospective study reported that in terms of long-term efficacy, the 3-year survival rates of PG and TG were similar ( $96 \%$ and $92 \%$, respectively), but the incidence of reflux esophagitis was significantly higher in the TG than PG group (14.5\% vs. 5.4\%; $P=0.02)$ [12]. Thus, after ensuring oncological safety, if we improve the PG approach to reduce the incidence of postoperative complications such as reflux esophagitis and anastomotic stenosis to a level similar to or even lower than that of TG, PG has the potential to become the standard surgical method for AEG.

Yamashita[13] found that double-tract reconstruction (DTR), which allows food to pass through the residual stomach into the duodenum, can effectively maintain postoperative nutritional status. However, some studies indicated that dietary bolus after DTR cannot enter the duodenum through the residual stomach as scheduled, rather escape through the jejunum route, which ultimately results in nutritional results similar to TG with Roux-en-Y reconstruction (TGRY) $[14,15]$. In addition, a retrospective study have recently demonstrated DTR and esophagogastrostomy has similar nutritional outcomes and QOL[16]. PJIRSTR completely blocked the passage of the proximal jejunum and avoided food escaping through the jejunum. Futhermore, When food passes through the duodenum, it is fully mixed with bile and pancreatic juice, and stimulates the secretion and release of hormones such as pancreatic secretin and cholecystokinin in the gastrointestinal tract, which is conducive to the digestion and absorption of food. Compared to jejunal interposition (JI), PJIRSTR, a technique similar to RY, is relatively easy to perform. In addition, when cancer of the remnant stomach is found, PJIRSTR can deal better with the remnant stomach than JI. In terms of the length of the interposed jejunum, Tokunaga suggested that it should be $<10 \mathrm{~cm}[17]$. Some scholars have suggested that the ideal length is $10-15 \mathrm{~cm}[18-20]$, otherwise, it may cause intestinal food stasis and inconvenience during postoperative endoscopy. However, according to our clinical experience, when the length of the interposed jejunum is $12-15 \mathrm{~cm}$, the above problems can be solved.

In this context, we speculated that if PJIRSTR could effectively solve long-term complications of postoperative reflux and anastomotic stenosis, it may become an attractive treatment method for Siewert II and III AEG with a diameter $<4 \mathrm{~cm}$. In our study, only one of the 33 patients (3\%) had Los Angeles grade $B$ reflux esophagitis as confirmed by endoscopy, and $2(6 \%)$ patients had Visick grade II reflux symptoms. The postoperative anti-reflux effect of this method is completely comparable with some anti-reflux surgery methods that have been reported so far. For example, the incidence of reflux esophagitis after side-overlap esophagogastrostomy (SOFY) was 10\%. However, the procedure requires an experienced surgeon to perform it under a laparoscope[21]. The incidence of reflux esophagitis after the double-flap technique (DFT) was $0 \%$, and the incidence of reflux symptoms was $10 \%[22]$. This technology has a good effect in preventing reflux esophagitis, but technical difficulties and the risk of anastomotic stenosis is relatively high. Our analysis showed that PJIRSTR has better anti-reflux effect than other techniques and may be related to the following two mechanisms that have a dual anti-reflux effect. First, side-to-side anastomosis was performed between the jejunum and the anterior wall of the remnant stomach, while keeping the anastomosis $3-5 \mathrm{~cm}$ away from the stump of the remnant stomach. This method of 
anastomosis formed an "artificial stomach fundus" structure, effectively blocking the food or digestive fluid from flowing into the esophagus. Second, is the anti-reflux effect of jejunum interposition; PJIRSTR completely blocked the passage of the proximal jejunum, making alkaline reflux esophagitis almost impossible.

In this study, we performed adjuvant chemotherapy for all patients whose postoperative pathology was not staged I at that time. Therefore, 23 of 33 patients underwent chemotherapy. The 3-year survival rate of patients was $90.9 \%$; we observed that all patients who died within 3 years after surgery had stage IVA disease in pathologic staging. However, 18 of 21 patients with pathological stages of stage III and IV survived for more than 3 years. Thirteen patients survived more than 5 years at the last follow-up and are still alive. There were two patients still alive $<5$ years from the last follow-up. These results suggested that the pathological stage may not be the most important factor affecting the survival period of patients and PG may be feasible for Siewert II and III AEG with a diameter $<4 \mathrm{~cm}$, but further strong clinical data are required for confirmation.

In our study, we performed PJIRSTR, which achieved satisfactory surgical and postoperative results. No death during operation or severe perioperative complications were recorded in any of the 33 patients. Both early postoperative complications( $(9 \%)$ and late complications $(6 \%)$ were low compared to DTR[23].For the postoperative nutritional status of the patients, all indicators decreased significantly within 3 months after surgery. Compared to the Phase I clinical stud, the nutritional indicators of the patients did not return to the preoperative level at postoperative 12 months, which may be related to the status of advanced tumors in some patients. In our study, the average weight loss was $13.5 \%, 11.8 \%$, and $10 \%$ at postoperative 3,6 , and 12 months, respectively. Difficulty maintaining weight is a typical feature after gastrectomy, which is connected with reduced stomach volume[23]. However, all the nutritional indicators have been rising after surgery.

There are several limitations to this study. First, this was a retrospective study prone to inherent bias. second, we performed a simple mean description of the patient's nutritional status indicators and did not use reliable clinical assessments for objective evaluation. Third, the sample size of the study is relatively small, and more multi-center, prospective clinical data support is needed to validate the findings. Last, we did not consider the influence of lymph node dissection on patients' survival period. However, we believe that although there were many methods of digestive tract reconstruction to prevent postoperative reflux, it is not necessary to establish a unified standard as long as the oncological safety and surgical results are guaranteed. Based on their own experience, surgeons can choose a surgical method with excellent postoperative outcomes that suits them. In addition, we believe that the current clinical research of AEG seems to have reached a bottleneck. Perhaps we should pay more attention to basic research and combine clinical research with basic research to bring the greatest benefits to patients.

In conclusion, we report here our novel reconstruction method after PG-PJIRSTR-that shows satisfactory outcomes after the operation. Most importantly, PJIRSTR effectively addressed the problems 
of postoperative reflux and reflux esophagitis and improved the postoperative quality of life of patients. However, multi-center and prospective randomized trials are needed to verify its clinical application value.

\section{Declarations}

\section{Acknowledgments}

Not applicable.

\section{Authors' contributions}

All authors contributed to the study conception and design. Material preparation, data collection and analysis were performed by Wanhong Zhang, Baoqing Ren and Ning Xu. Supervision: Jianhong Dong. Formal analysis and investigation: Liqiang Lei and Linjie Li. The first draft of the manuscript was written by Yuchao $\mathrm{Ma}$ and all authors commented on previous versions of the manuscript. All authors read and approved the final manuscript.

\section{Funding}

This work was supported by Key R\&D Projects in Shanxi Province Research Fund.(no. 201803D31167)

\section{Availability of data and materials}

The data used to support the findings of this study are available from the corresponding author upon request.

\section{Ethics approval and consent to participate}

This study was approved by the ethics committee of Shanxi Tumor Hospital. All patients signed informed consent.

\section{Consent for publication}

The patient was given his consent for information about himself to be published in World Journal of Surgical Oncology.

\section{Competing interests}

The authors declare that they have no competing interests

\section{References}

1. Runge TM, Abrams JA, Shaheen NJ. Epidemiology of Barrett's Esophagus and Esophageal Adenocarcinoma. Gastroenterol Clin North Am. 2015;44:203-31. 
2. Liu K, Yang K, Zhang W, Chen X, Chen X, Zhang B, et al. Changes of Esophagogastric Junctional Adenocarcinoma and Gastroesophageal Reflux Disease Among Surgical Patients During 1988-2012: A Single-institution, High-volume Experience in China. Ann Surg. 2016;263:88-95.

3. Colquhoun A, Arnold M, Ferlay J, Goodman KJ, Forman D, Soerjomataram I. Global patterns of cardia and non-cardia gastric cancer incidence in 2012. Gut. 2015;64:1881-8.

4. Yamasaki M, Takiguchi S, Omori T, Hirao M, Imamura H, Fujitani K, et al. Multicenter prospective trial of total gastrectomy versus proximal gastrectomy for upper third cT1 gastric cancer. Gastric Cancer. 2021;24:535-543.

5. Zhao L, Ling R, Chen J, Shi A, Chai C, Ma F, et al. Clinical Outcomes of Proximal Gastrectomy versus Total Gastrectomy for Proximal Gastric Cancer: A Systematic Review and Meta-Analysis. Dig Surg. 2021;38:1-13.

6. Rosa Fausto, Quero Giuseppe, Fiorillo Claudio et al. Total vs proximal gastrectomy for adenocarcinoma of the upper third of the stomach: a propensity-score-matched analysis of a multicenter western experience (On behalf of the Italian Research Group for Gastric Cancer-GIRCG). Gastric Cancer. 2018;21:845-852.

7. Yura M, Yoshikawa T, Otsuki S, Yamagata Y, Morita S, Katai H, et al. Oncological safety of proximal gastrectomy for T2/T3 proximal gastric cancer. Gastric Cancer. 2019;22:1029-1035.

8. Tao K, Dong JH. Phase I Clinical Research of Jejunal Interposition in Adenocarcinoma of the Esophagogastric Junction II/III Proximal Gastrectomy. Gastroenterol Res Pract. 2016;2016:1639654.

9. Japanese Gastric Cancer Association. Japanese gastric cancer treatment guidelines 2018 (5th edition). Gastric Cancer. 2021 Jan;24:1-21.

10. Sugoor P, Shah S, Dusane R, Desouza A, Goel M, Shrikhande SV. Proximal gastrectomy versus total gastrectomy for proximal third gastric cancer: total gastrectomy is not always necessary. Langenbecks Arch Surg. 2016;401:687-97.

11. Haruta S, Shinohara H, Hosogi H, Ohkura Y, Kobayashi N, Mizuno A, et al. Proximal gastrectomy with exclusion of no. $3 \mathrm{~b}$ lesser curvature lymph node dissection could be indicated for patients with advanced upper-third gastric cancer. Gastric Cancer. 2017;20:528-535.

12. Yamasaki M, Takiguchi S, Omori T, Hirao M, Imamura H, Fujitani K, et al. Multicenter prospective trial of total gastrectomy versus proximal gastrectomy for upper third cT1 gastric cancer. Gastric Cancer. 2021;24:535-543.

13. Yamashita K, Iwatsuki M, Koga Y, Toihata T, Kiyozumi Y, Kuroda D, et al. Preservation of physiological passage through the remnant stomach prevents postoperative malnutrition after proximal gastrectomy with double tract reconstruction. Surg Today. 2019;49:748-754.

14. Tanaka K, Ebihara Y, Kurashima Y, Nakanishi Y, Asano T, Noji T, et al. Laparoscopic proximal gastrectomy with oblique jejunogastrostomy. Langenbecks Arch Surg. 2017;402:995-1002.

15. Cho M, Son T, Kim HI, Noh SH, Choi S, Seo WJ, et al. Similar hematologic and nutritional outcomes after proximal gastrectomy with double-tract reconstruction in comparison to total gastrectomy for early upper gastric cancer. Surg Endosc. 2019 Jun;33:1757-1768. 
16. Tominaga S, Ojima T, Nakamura M, Katsuda M, Hayata K, Kitadani J, et al. Esophagogastrostomy With Fundoplication Versus Double-tract Reconstruction After Laparoscopic Proximal Gastrectomy for Gastric Cancer. Surg Laparosc Endosc Percutan Tech. 2021 May 11;31(5):594-598.

17. Tokunaga M, Ohyama S, Hiki N, Hoshino E, Nunobe S, Fukunaga T, et al. Endoscopic evaluation of reflux esophagitis after proximal gastrectomy: comparison between esophagogastric anastomosis and jejunal interposition. World J Surg. 2008;32:1473-7.

18. Aburatani T, Kojima K, Otsuki S, Murase H, Okuno K, Gokita K, et al. Double-tract reconstruction after laparoscopic proximal gastrectomy using detachable ENDO-PSD. Surg Endosc. 2017;31:4848-4856.

19. Ko HJ, Kim KH, Lee SH, Choi CW, Kim SJ, In Choi C, et al.Can Proximal Gastrectomy with DoubleTract Reconstruction Replace Total Gastrectomy? A Propensity Score Matching Analysis. J Gastrointest Surg. 2020;24:516-524.

20. Nomura E, Lee SW, Kawai M, Yamazaki M, Nabeshima K, Nakamura K, et al. Functional outcomes by reconstruction technique following laparoscopic proximal gastrectomy for gastric cancer: double tract versus jejunal interposition. World J Surg Oncol. 2014;12:20.

21. Yamashita Y, Yamamoto A, Tamamori Y, Yoshii M, Nishiguchi Y. Side overlap esophagogastrostomy to prevent reflux after proximal gastrectomy. Gastric Cancer. 2017;20:728-735.

22. Kuroda S, Nishizaki M, Kikuchi S, Noma K, Tanabe S, Kagawa S, et al. Double-Flap Technique as an Antireflux Procedure in Esophagogastrostomy after Proximal Gastrectomy. J Am Coll Surg. 2016;223:e7-e13.

23. Ahn SH, Jung DH, Son SY, Lee CM, Park DJ, Kim HH. Laparoscopic double-tract proximal gastrectomy for proximal early gastric cancer. Gastric Cancer. 2014;17(3):562-70.

24. Katai H, Sano T, Fukagawa T, Shinohara H, Sasako M. Prospective study of proximal gastrectomy for early gastric cancer in the upper third of the stomach. Br J Surg. 2003;90:850-3.

\section{Tables}

\section{Table 1 Patient demographics}


PJIRSTR $(n=\quad$ Range

33)

Age (years, mean $\pm S D$ )

$60.9( \pm 11.9)$

46-72

$<60$

$12 \rrbracket 36.3 \% \rrbracket$

$\geq 60$

$21 \llbracket 63.6 \% 区$

Gender (male : female)

29:4

Smoking

11(33.3\%)

Drinking

$4(12.1 \%)$

Body mass index (BMI, kg/m2)

$24.1( \pm 2.6)$

$18.25-$

28.26

Previous abdominal surgery

$0(0 \%)$

Comorbidity

1. High blood pressure

$5(15.2 \%)$

2.Diabetes mellitus

$3(9.1 \%)$

3.Cardiovascular disease

$1(3 \%)$

ASA score

I

$5(15.2 \%)$

II

$27(81.8 \%)$

III

$1(3 \%)$

Siewert type

II

$23(69.7 \%)$

III

$10(30.3 \%)$

Gastroesophageal reflux symptoms(based on preoperative Visick

$3(9.1 \%)$ score》

Reflux esophagitis

$0(0 \%)$

(based on the preoperative evaluation of endoscopy)

Table 2 Surgical outcomes and complications 


\begin{tabular}{lll} 
& Values & Range \\
\hline Operative time (minutes) & $136.7 \pm 22.4$ & $110-180$ \\
\hline Digestive tract reconstruction time (min) & $32.3 \pm 5.0$ & $26-45$ \\
\hline Lymph node dissection(n,\%) & & \\
\hline D1+ & $4(12.1 \%)$ & \\
\hline D2 & $29(87.9 \%)$ & \\
\hline R0 resection (n, \%) & $33(100 \%)$ & \\
\hline Intraoperative blood loss (ml) & $87.6 \pm 18.1$ & $60-120$ \\
\hline Combined resection (n) & & \\
\hline Gall bladder & 3 & \\
\hline Spleen & 1 & \\
\hline First anal exhaust time (days) & $2.7 \pm 1.2$ & \\
\hline Postoperative hospital stays (days) & $7.5 \pm 1.2$ & $6-10$ \\
\hline Median follow-up time (months) & 60.7 & $27-90.8$ \\
\hline Early complications (n) & & \\
\hline Anastomotic bleeding & 1 & \\
\hline Incision infection & 1 & \\
\hline Ileus & 0 & \\
\hline Anastomotic stenosis & 0 & \\
\hline Organ dysfunctions & & \\
\hline Leakage of anastomosis & & \\
\hline Late complications & & \\
\hline Reflux esophagitis(n, \%) & & \\
\hline Reflux symptom(n, \%) & & \\
\hline Recannulation of intestinal tract(n, \%) & & \\
\hline
\end{tabular}

\section{Table 3 Pathologic findings}




\begin{tabular}{|c|c|c|}
\hline & PJIRSTR(n=33) & Range \\
\hline Lymph nodes dissection number $(\mathrm{n})$ & $14.7 \pm 5.1$ & $9-27$ \\
\hline Number of metastatic lymph nodes & $2.6 \pm 3.4$ & $0-12$ \\
\hline Tumor size $(\mathrm{cm})$ & $3.06 \pm 0.84$ & $1-4$ \\
\hline \multicolumn{3}{|l|}{ Differentiation } \\
\hline High-middle & $21(63.6 \%)$ & \\
\hline Low & $12(36.3 \%)$ & \\
\hline \multicolumn{3}{|l|}{ T stage } \\
\hline T1a & 3 & \\
\hline $\mathrm{T} 1 \mathrm{~b}$ & 3 & \\
\hline $\mathrm{T} 2$ & 7 & \\
\hline T3 & 4 & \\
\hline $\mathrm{T} 4 \mathrm{a}$ & 16 & \\
\hline \multicolumn{3}{|l|}{$\mathrm{N}$ stage } \\
\hline NO & 14 & \\
\hline N1 & 6 & \\
\hline N2 & 8 & \\
\hline N3 & 5 & \\
\hline \multicolumn{3}{|l|}{ Stage } \\
\hline IA & 3 & \\
\hline IB & 2 & \\
\hline IC & 5 & \\
\hline IIB & 2 & \\
\hline IIIA & 1 & \\
\hline IIIB & 8 & \\
\hline IVA & 12 & \\
\hline Adjuvant chemotherapy(n,\%) & $23(69.7 \%)$ & \\
\hline
\end{tabular}


Table 4 Comparison between Phase I clinical research and current study in our institution

\begin{tabular}{llll} 
& $\begin{array}{l}\text { Phase I } \\
\text { research }(\mathrm{n}=15)\end{array}$ & $\begin{array}{l}\text { Current } \\
\text { study }(\mathrm{n}=33)\end{array}$ & $P$ \\
\hline $\begin{array}{l}\text { Digestive tract reconstruction } \\
\text { time(minutes) }\end{array}$ & $29.5 \pm 5.7$ & $32.3 \pm 5.0$ & $>0.05$ \\
\hline Intraoperative blood loss $(\mathrm{ml})$ & $96.7 \pm 20.2$ & $87.6 \pm 18.1$ & 0.841 \\
\hline Postoperative hospital stays (days) & $10.7 \pm 2.3$ & $7.5 \pm 1.2$ & 0.012 \\
\hline Early complications & $7 \%(\mathrm{n}=1)$ & $9 \%(\mathrm{n}=3)$ & 1 \\
\hline Anastomotic stenosis & $0 \%(\mathrm{n}=0)$ & $0 \%(\mathrm{n}=0)$ & \\
\hline Reflux symptoms & & & \\
\hline Visick I & 14 & 0 & \\
\hline Visick II & 0 & 2 & \\
\hline Visick III & 1 & 0 & \\
\hline Reflux esophagitis & 12 & 0 & \\
\hline Grade A & 2 & 1 & \\
\hline Grade B & 1 & 0 & \\
\hline Grade C & & & \\
\hline
\end{tabular}

Figures 
A

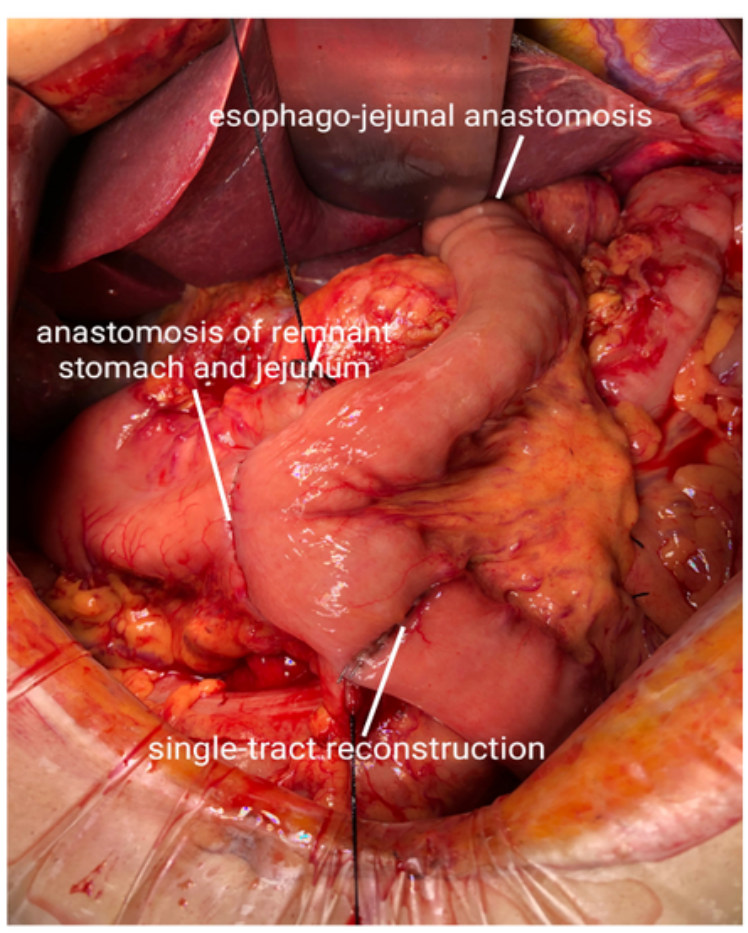

B

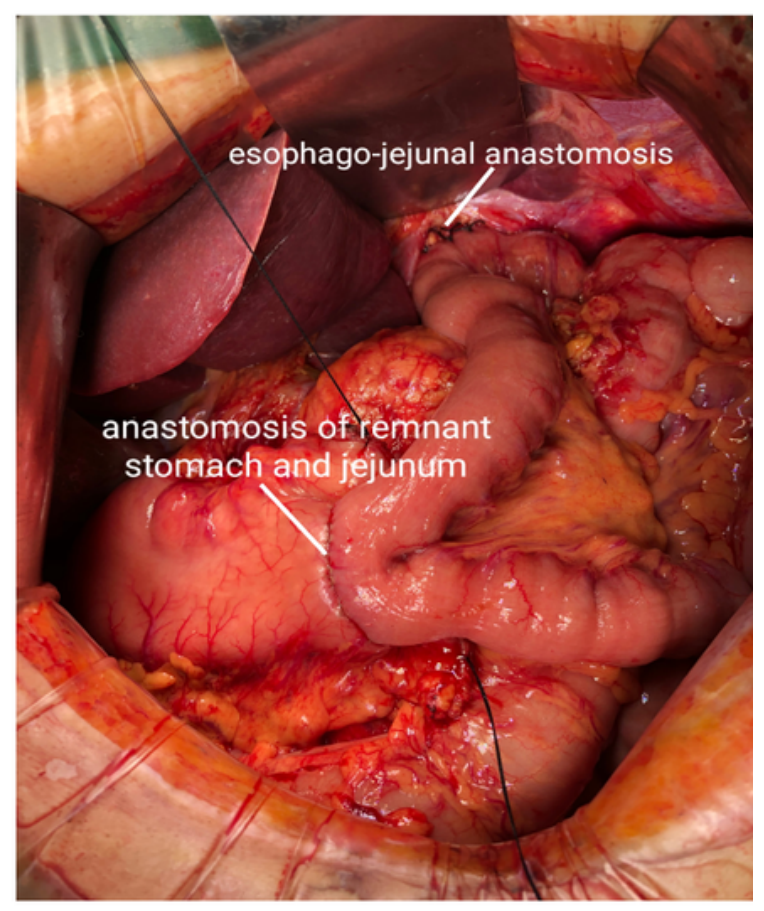

$\mathrm{C}$

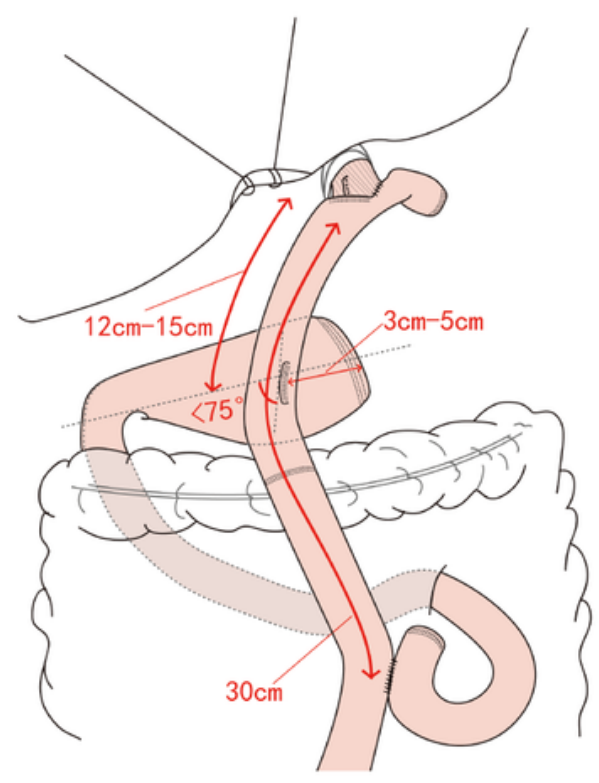

\section{Figure 1}

The piggyback jejunal interposition reconstruction single-tract reconstruction (PJIRSTR). (a) is the picture of the PG with PJIRSTR; (b) is the picture when the jejunum is not blocked (c) is the schematic diagram. 


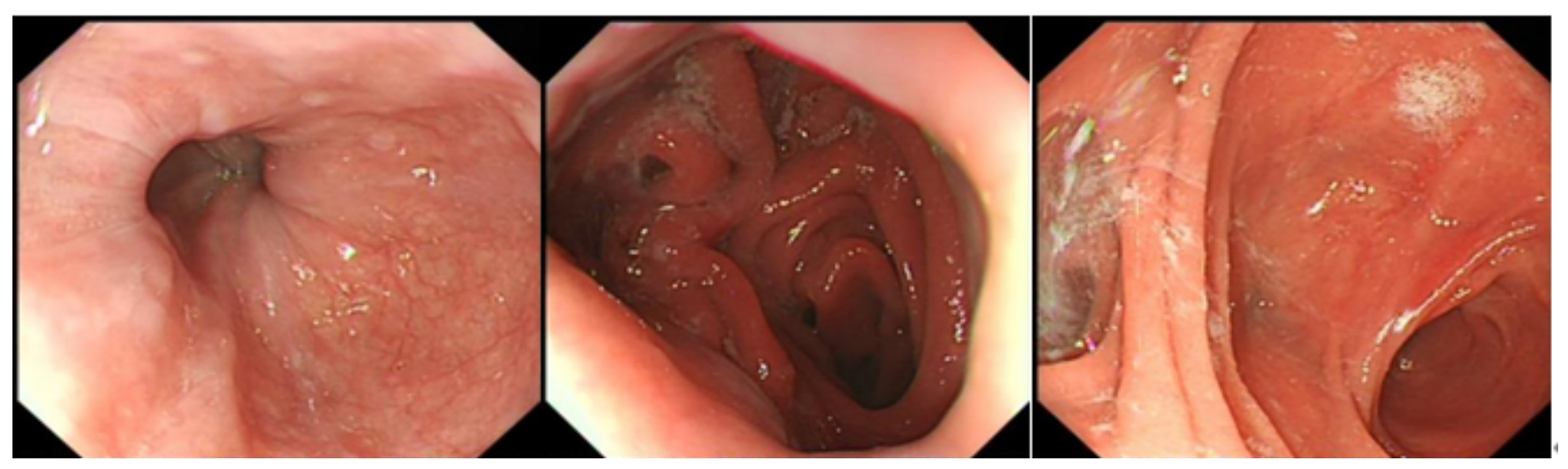

Figure 2

Gastroscopic examination after the operation.

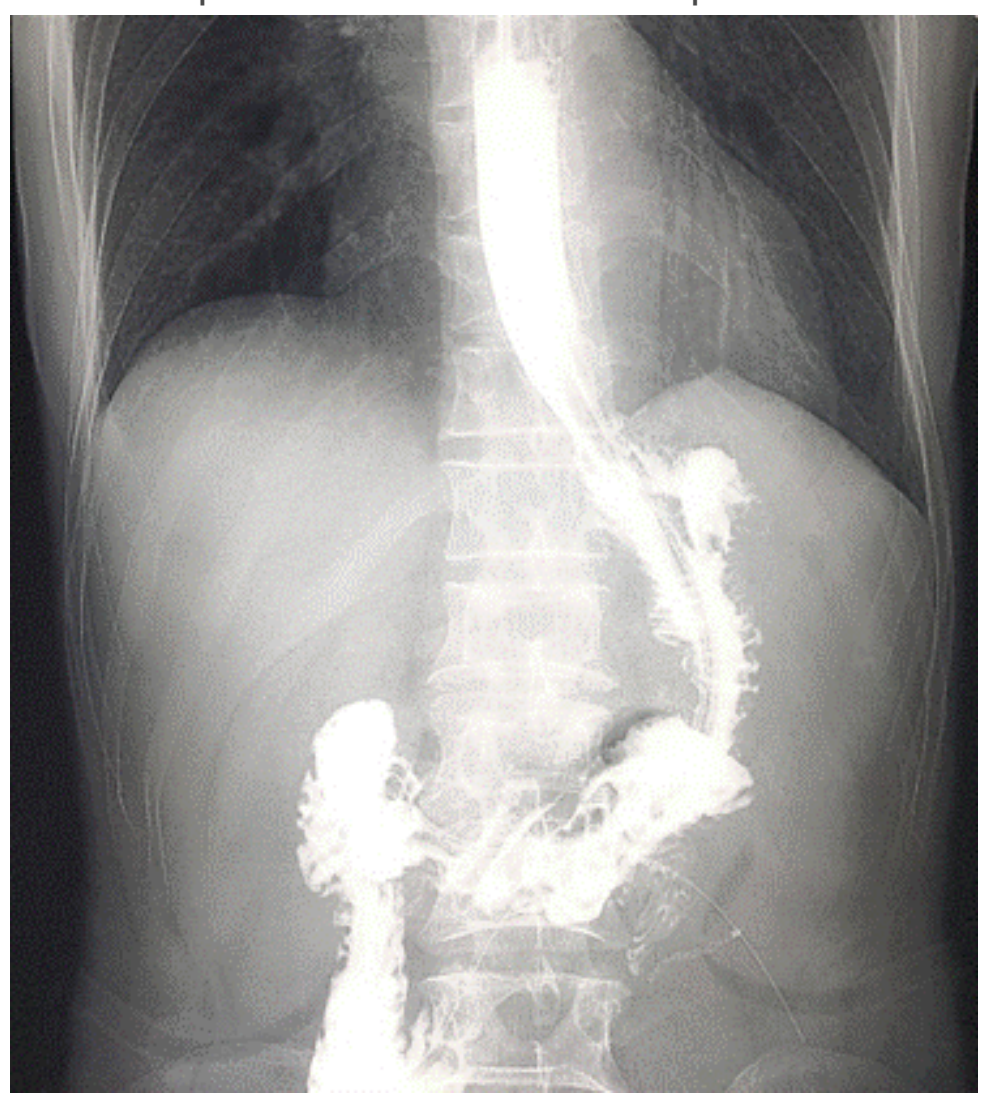

Figure 3

The upper gastrointestinal radiography findings after PG with PJIRSTR. The contrast medium flowing from the esophagus into the remnant stomach was extremely good. 
A

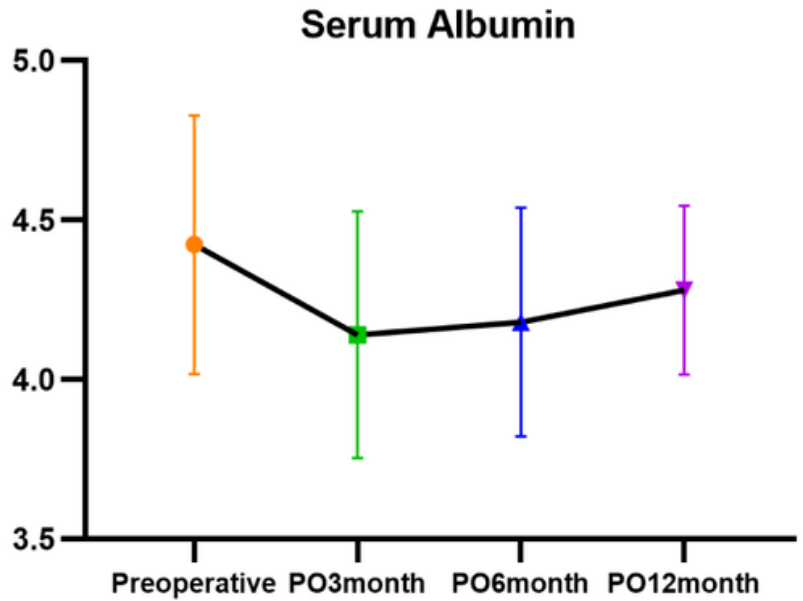

C

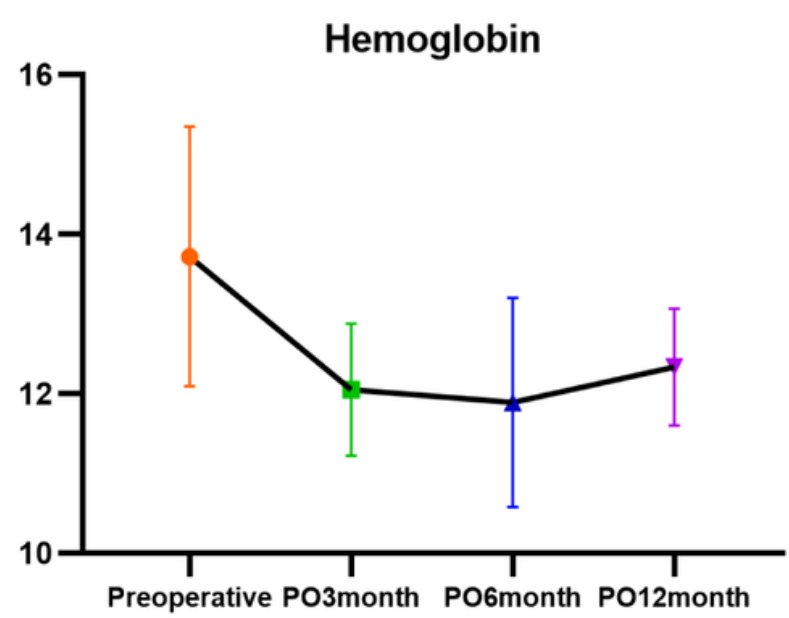

B

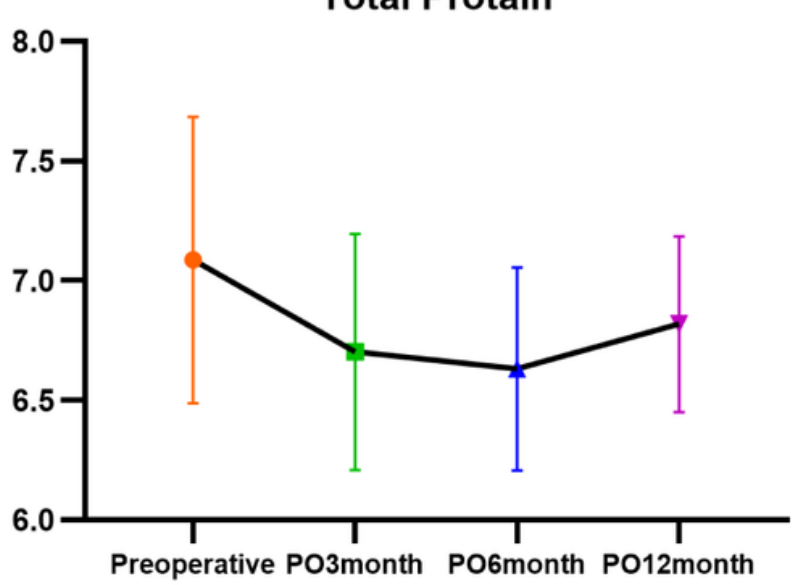

$\mathrm{D}$

Weight

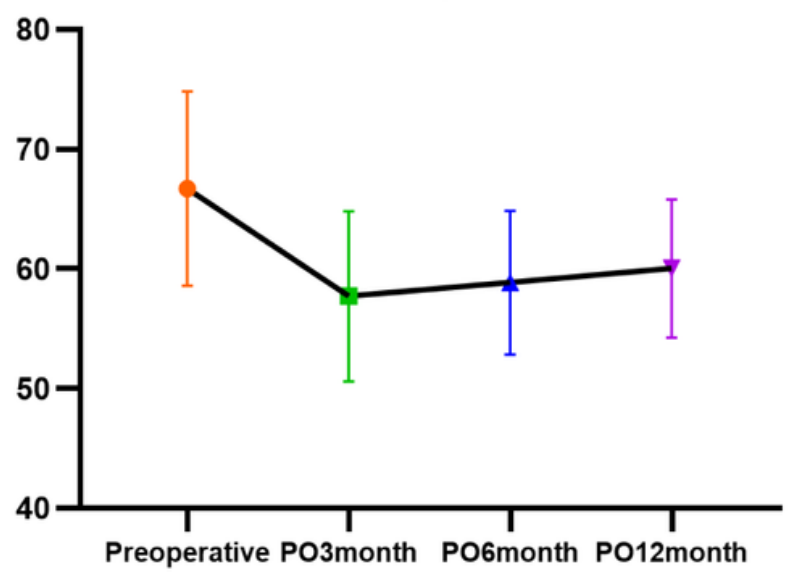

Figure 4

Changes in nutritional indicators. (a) is the picture of the changes of serum albumin; (b) is the picture of the changes of total protein; (c) is the picture of the changes of hemoglobin; (d) is the picture of the changes of weight. 